\title{
Synthesis of hydroxyapatite nanopowders by sol-gel emulsion technique
}

\author{
K SARANYA, MEENAL KOWSHIK and SUTAPA ROY RAMANAN* \\ Birla Institute of Technology and Science, Pilani-Goa Campus, Zuarinagar, Goa 403 726, India
}

MS received 7 July 2009; revised 21 February 2011

\begin{abstract}
Hydroxyapatite nanopowders were synthesized by a sol-gel emulsion technique by varying the concentration of a non-ionic surfactant in the organic phases (oil phase) of water-in-oil (w/o) emulsion. Calcium acetate dissolved in distilled water and phosphorous pentoxide dissolved in 2-butanol were used as starting precursors. The prepared sol was emulsified in a support solvent (cyclohexane) containing 2,4 and 5 volume\% of surfactant (Span 80), followed by the addition of triethylamine, for gelation. The gel powders thus obtained were calcined at different temperatures up to $750^{\circ} \mathrm{C}$. Characterization was done using XRD, SEM and TEM. Pellets were made from the developed HAP powders and tested for its biocompatibility after their immersion in the simulated body fluid.
\end{abstract}

Keywords. Hydroxyapatite; sol-gel preparation; nanoparticles; emulsion; microstructure.

\section{Introduction}

Hydroxyapatite $\mathrm{Ca}_{10}\left(\mathrm{PO}_{4}\right)_{6}(\mathrm{OH})_{2}$ (HAP), is one of the most attractive biomaterials used for bone implant due to its compositional and biological similarity to the native tissues of human beings (Hench 1998). It is widely used in both orthopedic and dental fields due to its chemical similarity to bone mineral, excellent biocompatibility, tissue bioactivity and capability of bonding directly to bone, thus allowing for true osteointegration (Hing et al 1999; Oonishi et al 1999; Chavan et al 2010).

HAP crystallites in the human bone are so small that they are considered to be in the nano regime. The poor mechanical strength and load bearing ability of synthesized HAP bodies has so far limited its use in replacements ( $\mathrm{Li}$ 2008). Nanopowders due to their high surface reactivity are expected to improve the densification and hence improve the strength. It has also been seen to have better bioactivity (Zamami et al 2008). As a result, extensive efforts are on to synthesize uniform and well-sintered HAP bodies starting from nanopowders. Several methods have been used in synthesis of nano-HAP, such as chemical precipitation (Sung et al 2004; Loo et al 2008), nanoemulsion synthesis (Zhou et al 2008), micelle-templated synthesis (Wu and Bose 2005; Coelho et al 2010), hydrothermal reaction, porous microgels (Schachschal et al 2008; Du et al 2009), mechanochemical synthesis (Mochales et al 2004; Tabrizi et al 2009), microwave synthesis (Liu et al 2005) and sol-gel synthesis (Han et al 2004; Zamami et al 2008). Sol-gel method has the advantage that it

\footnotetext{
*Author for correspondence (sutapa @ bits-goa.ac.in)
}

offers a molecular-level mixing of the calcium and phosphorous precursors, thus improving chemical homogeneity and reducing formation temperature of resulting HAP. Moreover, it does not require long experimentation time and sophisticated instrumentation (Liu et al 2002; Ramanan and Ramanan 2002; Weng et al 2003).

In this work we have prepared HAP nanopowders by solgel emulsion technique, where the sol (water medium) is emulsified, in a support solvent (oil medium) in the presence of a surfactant, into droplets by continuous stirring. Gelation of the sol droplets is achieved by adding gelling agent and maintaining the desired $\mathrm{pH}$. This paper reports the synthesis and characterization of these HAP nanopowders.

\section{Materials and methods}

\subsection{Sol preparation}

The sol was prepared by mixing a $2 \mathrm{M}$ solution of calcium acetate dissolved in distilled water with $1 \mathrm{M}$ solution of phosphorous pentoxide dissolved in 2-butanol under continuous stirring. Acetic acid was added into calcium acetate solution to prevent the immediate precipitation and to improve the miscibility between the two solutions. The $\mathrm{Ca} / \mathrm{P}$ atomic ratio was maintained at 1.67 . The homogenous sol was heated at $80^{\circ} \mathrm{C}$ to reduce the volume to half of its initial volume, so as to obtain a concentrated sol.

\subsection{Emulsion-gel process}

Cyclohexane was used as the support solvent (oil medium). The desired amount of surfactant (Span 80) 

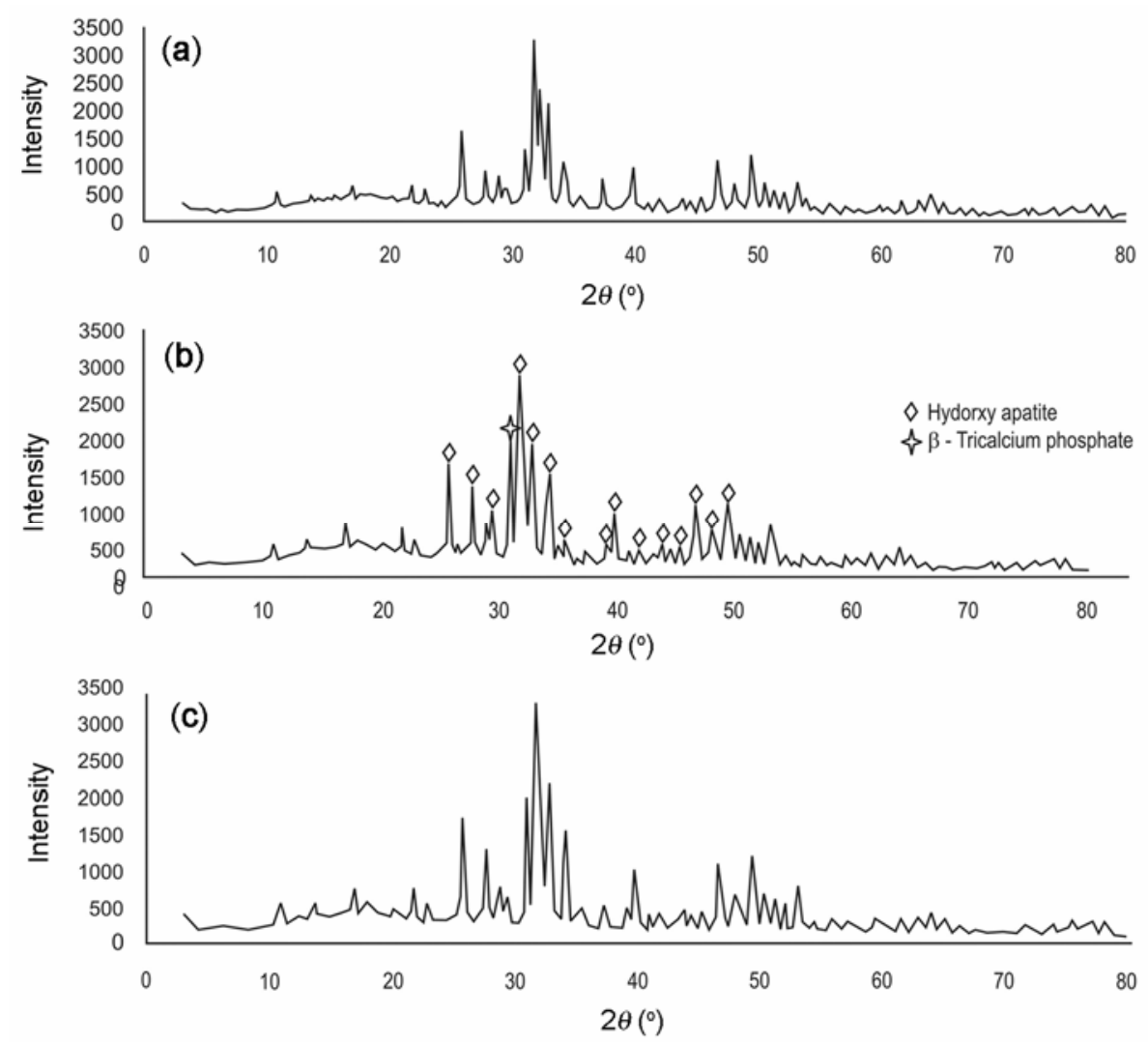

Figure 1. XRD patterns for the hydroxyapatite nanoparticles synthesized using (a) $2 \%$, (b) $4 \%$ and (c) $5 \%$ surfactants.

which was varied as 2,4 and $5 \%$ by volume of the support solvent was added to it. The sol : support solvent ratio was maintained at $1: 3$. The concentrated sol was added in drop-wise manner into the support solvent, under continuous stirring to form water in oil type emulsion. Stirring was continued for $10 \mathrm{~min}$ after completion of addition of sol. The sol (water medium) forms immiscible droplets in the support solvent (oil medium) under continuous stirring. The surfactant helps to form these micellar structures and retain their shapes. Triethylamine was added to the emulsion as a gelling agent. The addition was continued until a $\mathrm{pH}$ of 9.0 was achieved to ensure complete gelation. The resulting gel particles were separated from the solvent by filtration, washed thrice with acetone, filtered and air dried. The powder obtained was further dried in the oven at $80^{\circ} \mathrm{C}$ for 2 days and crushed using a mortar and pestle. The fine powder obtained was calcined at a temperature of up to $750^{\circ} \mathrm{C}$ using carbolite furnace at the rate of $4{ }^{\circ} \mathrm{C}$ per minute. The soaking time at the final temperature was $5 \mathrm{~h}$.

\subsection{HAP pellet preparation}

The pellets were prepared by mixing the calcined HAP powder with distilled water $(7 \%)$ and pressing $6 \mathrm{~g}$ of the powder using a punch and die set and a $\mathrm{KBr}$ press (model
M-15) with a pressure of 2 ton. The pellets were then heat treated at $500^{\circ} \mathrm{C}$ for proper densification.

\subsection{Immersion in simulated body fluid (SBF)}

The SBF solution was prepared as described by Cuneyt Tas (2000). The HAP pellets were soaked under static condition in $100 \mathrm{ml}$ of SBF at $37^{\circ} \mathrm{C}$ in a water bath. For characterization studies, the pellets were removed from the SBF, gently washed with distilled water and dried in an air oven at $100^{\circ} \mathrm{C}$ for $4 \mathrm{~h}$.

\subsection{Characterization}

The calcined HAP powders were characterized using X-ray diffraction (XRD) (Rigaku Miniflex) from $3^{\circ}$ to $70^{\circ}$ at a scan speed of $0.1^{\circ} 2 \theta / \mathrm{min}$. The crystallite size of the calcined powder was measured using Scherer's equation, $D=k \lambda / B \cos \theta$; where $D$ is the crystallite diameter in $\AA, k$ the shape constant $(0 \cdot 9), \lambda$ the X-ray $\left(\mathrm{CuK}_{\alpha}\right)$ wavelength in $\AA, \theta$ the diffraction angle in degrees and $B$ (in radian) the half width measured for the XRD peak. The microstructures were studied using transmission electron microscopy (TEM) (Phillips CM 200) and scanning electron microscopy (SEM) (JEOL JSM 6360 LV). 


\section{Results and discussions}

\section{$3.1 X R D$}

Figure 1 shows the XRD patterns of HAP powder calcined at $750^{\circ} \mathrm{C}$, for 2,4 and $5 \%$ of surfactant concentrations. Sharp crystalline phases of HAP are present in all the cases. The HAP was polycrystalline and $\beta$-tricalcium phosphate $\left(\beta\right.$-TCP) as whitlockite $\left(\mathrm{Ca}_{3}\left(\mathrm{PO}_{4}\right)_{2}\right)$ was present in trace amounts (table 1$)$. Presence of small amount of $\beta$-TCP along with HAP has been reported to be formed due to the thermal decomposition of HAP (Weng and Baptista 1998; Rhee 2002; Han et al 2004). $\alpha$ - and $\beta$-tricalcium phosphate phases are highly bioresorbable and presence of small amount of $\beta$-TCP in HAP would be helpful for the strong and fast bonding of the artificial bones to natural ones via rapid dissolution. However, high content of $\beta$-TCP would cause too much resorption in human body, resulting in serious strength degradation in artificial bone replacements (Sung et al 2004).

The volume fraction of $\beta$-TCP was estimated using the formula (Sung et al 2004)

$$
X_{\beta}=P W_{\beta} /\left[1+(P-1) W_{\beta}\right],
$$

where

$$
W_{\beta}=I_{\beta} /\left(I_{\beta}+I_{H}\right),
$$

$I_{\beta}$ and $I_{H}$ are the XRD intensity values of $\beta$-TCP [ $\left[\begin{array}{lll}0 & 2 & 10\end{array}\right]$ and HAP [2 111 ] reflections, respectively, for $2 \theta=30.9^{\circ}$ and $31.7^{\circ}$. In this, the coefficient $P$, representing the integrated intensity ratio of HAP reflection to $\beta$-TCP reflection, was $2 \cdot 275$ as determined by Sung et al (2003), using $\mathrm{XRD}$ on the mixtures of standard HAP and $\beta$-TCP powders. The volume fraction of $\beta$-TCP was found to be in the range of $0.47-0 \cdot 6$ (table 1 ).

The crystallite size calculated using the peak corresponding to $2 \theta=31.7^{\circ}$ varied between 23 and $37 \mathrm{~nm}$ without showing any specific trend. In an emulsion, when the surfactant concentration reaches or just exceeds critical micellar concentration (CMC), the droplet size becomes significantly small due to the reduction in interfacial tension resulting in dispersion of large droplets into smaller ones (Bourrel and Schechter 1988; Ganguli and Chatterjee 1997; Siladitya et al 1999). At or above $\mathrm{CMC}$, the spherical micelles enclose the sol in water

Table 1. Comparison of crystallite size and the volume percentage of $\beta$-TCP for different surfactant concentrations after calcinations at $750^{\circ} \mathrm{C}$.

\begin{tabular}{lccc}
\hline Surfactant concentration $(\%)$ & 2 & 4 & 5 \\
Crystallite size $(\mathrm{nm})$ & $30 \cdot 00$ & $37 \cdot 00$ & $23 \cdot 74$ \\
Percentage of $\beta$-TCP & $0 \cdot 4708$ & $0 \cdot 5862$ & $0 \cdot 601$ \\
Particle size (nm) & $22-40$ & $30-35$ & $18-25$ \\
\hline
\end{tabular}

pools. The size of the pool varies with the aggregation number of surfactant molecules in the micelle and the relative quantity of water phase (Bourrel and Schechter 1988). Micelles in water-in-oil systems are known to have much smaller aggregation number than those in oil-in-water system leading to relatively smaller


Figure 2. Typical TEM micrographs of HAP nanoparticles synthesized using (a) 2\%, (b) $4 \%$ and (c) $5 \%$ surfactant concentrations. 
water pools (Bourrel and Schechter 1988). For surfactant concentrations exceeding the $\mathrm{CMC}$, the droplets are known to rearrange themselves in different shapes (Bourrel and Schechter 1988; Ramsay 1996; Behrens et al 1997; Borisch et al 1998).

\subsection{TEM}

Figure 2 shows the TEM micrographs. It is observed that at $2 \%$ surfactant concentration (figure $2 \mathrm{a}$ ), the particles were mostly hexagonal in shape, showing well-defined edges. The particle size was in the range of $22-40 \mathrm{~nm}$; with most of the particles averaging at around $30 \mathrm{~nm}$. At $4 \%$ surfactant concentration (figure $2 \mathrm{~b}$ ) most of the particles were in the size range of $30-35 \mathrm{~nm}$ with a few smaller particles of $12-15 \mathrm{~nm}$ embedded between them. The particles were mostly irregular in shape without any well-defined outline. When $5 \%$ of surfactant (figure 2c) was used, the particles were mostly spherical and 18 $24 \mathrm{~nm}$ in size. Few particles of $\sim 40 \mathrm{~nm}$ were also observed. Surfactant concentrations above CMC have been reported to show a transformation from spherical to

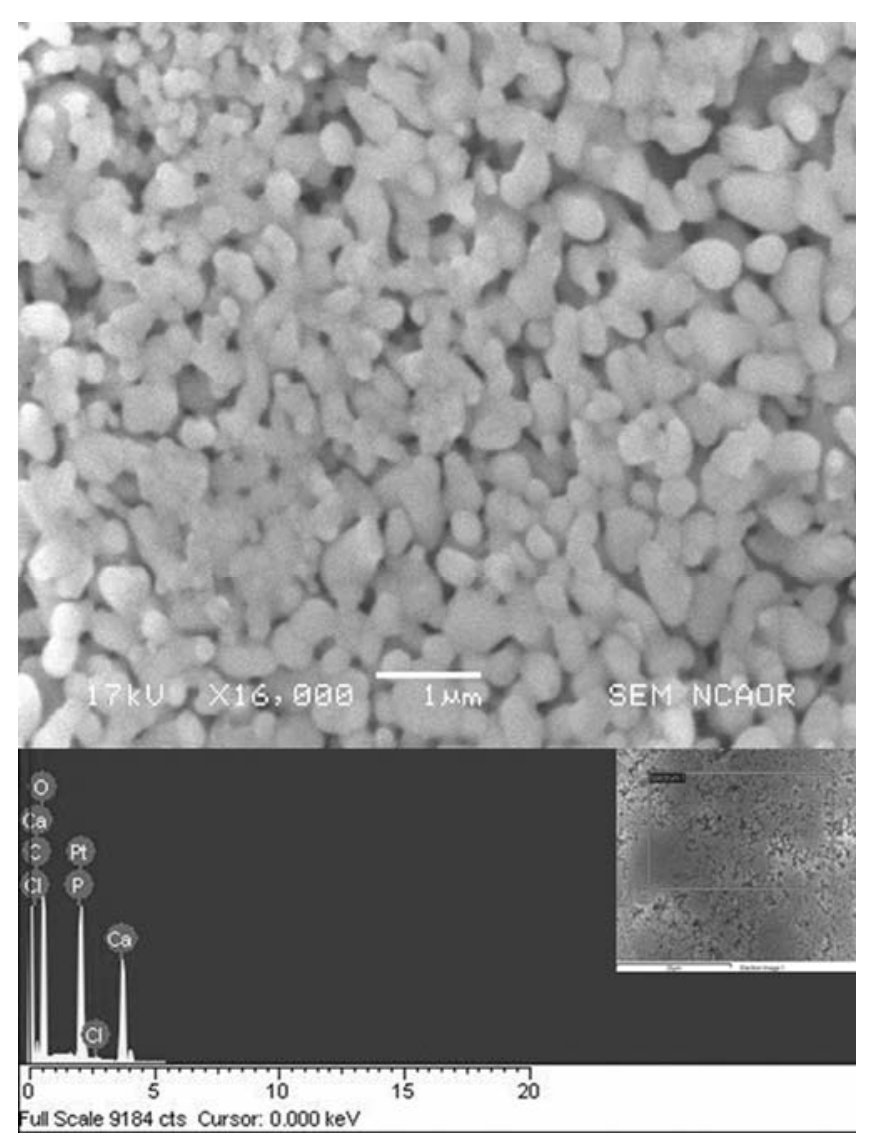

Figure 3. (a) The SEM image of the HAP bodies after immersion in SBF for 1 month. (b) EDAX spectrum of the same HAP body (inset in figure $3 \mathrm{~b}$ shows the region from where the EDAX spectrum was recorded). non-spherical shapes (Bourrel and Schechter 1988). However in the present case, more or less spherical particles were observed for the highest surfactant concentration which may be attributed to the fact that increased surfactant concentration facilitates the possibility of the droplets remaining in their minimum surface tension configuration.

\subsection{HAP pellets}

HAP pellets were immersed in SBF for 1 month at physiological conditions ( $\mathrm{pH} 7.4$ and $37^{\circ} \mathrm{C}$ ) and subsequently characterized using XRD and SEM. The XRD pattern showed no significant change of phase. Similar observation has been reported by Dorozkhina et al (2009) for pellets made from commercially available HAP and laboratory synthesized HAP, wherein there was no change in the XRD pattern after incubation in SBF for 20 days. The SEM micrograph (figure 3), shows the presence of porous network structure which is desirable for the formation of apatite layer on the surface due to a sequence of chemical reactions like precipitation, nucleation and growth of calcium phosphate (Cai et al 2009; Chavan et al 2010). Such a structure would be suitable for the intergrowth of natural and artificial bones. The EDAX spectrum of a selective area on the pellet showed the presence of $\mathrm{Ca}, \mathrm{P}$ and $\mathrm{O}$, confirming no drastic changes in the composition of the HAP pellet. Signals of $\mathrm{Pt}$ and $\mathrm{C}$ were observed due to the coating material and the substrate.

\section{Conclusions}

Hydroxyapatite nanopowders have been successfully produced by a sol-gel emulsion technique at a relatively low temperature using calcium acetate solution in water and acetic acid and phosphorous pentoxide in 2-butanol. Water in oil emulsion was formed by dispersing the sol in a support solvent of cyclohexane with varying amounts of Span 80. XRD confirmed the presence of crystalline hydroxyapatite along with a small amount of $\beta$-TCP varying between 0.47 and $0.6 \%$. The crystallite sizes of the HAP powders varied between 23 and $37 \mathrm{~nm}$ and the particles size was obtained between 20 and $40 \mathrm{~nm}$. The shape of the particles changed from hexagonal to spherical with increase in surfactant concentration. Hydroxyapatite pellets immersed in SBF for 1 month showed no changes in crystallinity and microstructure.

\section{Acknowledgements}

The authors would like to thank Dr Rahul Mohan, NCAOR, Goa, for helping with the SEM measurements and SAIF at IIT Mumbai for the TEM analysis. 


\section{References}

Behrens P, Glaue A, Haggenmuller C and Schechner G 1997 Solid State Ionics 101255

Borisch P, Diele S, Goring P, Kresse $\mathrm{H}$ and Tschierske C J 1998 Mater. Chem. 8529

Bourrel M and Schechter R S 1988 Microemulsions and related systems (New York: Marcel Dekker)

Cai Y, Zhang S, Zeng X, Wang Y, Qian M and Weng W 2009 Thin Solid Films 5175347

Chavan P N and Manjushri M Bahiretal 2010 Mater. Sci. Eng. B168 224

Coelho J, Meorira J, Almeida F and Monteiro J 2010 J. Mater Sci. Mater. Med. 212543

Cuneyt Tas A 2000 Biomaterials 211429

Dorozhkina S V 2009 J. Mater. Sci. 442343

Du X, Chu Y, Xing S and Dong L 2009 J. Mater. Sci. 446273

Ganguli D and Chatterjee M 1997 Ceramic powder preparation: a handbook (Boston: Kluwer Academic Publishers)

Han Y, Li S, Wang X and Chen X 2004 Mater. Res. Bull. 3925

Hench L L 1998 J. Am. Ceram. Soc. 811705

Hing K A, Best S M, Tanner K E, Bonfield W and Revell P A 1999 J. Mater. Sci. Mater. Med. 10663

Li C 2008 Powder Technol. doi 10.1016/j.powtec.2008.11.001

Liu D M, Troczynski T and Tseng W J 2002 Biomaterials 23 1227

Liu J, Li K, Wang H, Zhu M, Xu H and Yan H 2005 Nanotechnology 1682
Loo S C J, Siew Y E, Ho S, Boey F Y C and Ma J $2008 J$. Mater. Sci. Mater. Med. 191389

Mochales C, Briak-BenAbdeslam, Ginebra M P, Terol A, Planell J A and Boudeville P 2004 Biomaterials 251151

Oonishi H, Hench L L, Wilson J, Sugihara F, Tsuji E, Kushitani $\mathrm{S}$ and Iwaki H 1999 J. Biomed. Mater. Res. 4431

Ramanan S R and Ramanan V 2002 Asian J. Sci. Technol. 19 55

Ramsay J D F 1996 Sol-gel processing of advanced ceramics (ed.) F D Gnanam (New Delhi: Oxford \& IBH Publishing Co Pvt. Ltd)

Rhee S H 2002 Biomaterials 231147

Schachschal S, Pich A and Alder H J 2008 Langmuir 24 5129

Siladitya B, Chatterjee M and Ganguli D J 1999 Sol-Gel Sci. Tech. 16143

Sung Y M, Lee J C and Yang J W 2004 J. Cryst. Growth 262 467

Tabrizi B N, Honarmandi P, Kahrizsangi R E and Honarmandi P 2009 Mater. Lett. 63543

Weng W and Baptista JL 1998 Biomaterials 19125

Weng W, Zhang S, Cheng K, Qu H, Du P, Shen G, Yuan J and Han G 2003 Surf. Coat. Technol. 167292

$\mathrm{Wu} \mathrm{Y}$ and Bose S 2005 Langmuir 213232

Zamami A H R, Behnamghader A and Kazemzadeh A 2008 Mater. Sci. Eng. C28 1326

Zhou W Y, Wang M, Cheung W L, Guo B C and Jia D M 2008 J. Mater. Sci. Mater. Med. 19103 